REVISTA MATEMATICA COMPLUTENSE

Volumen 11, número 1: 1998

http://dx.doi.org/10.5209/rev_REMA.1998.v11.n1.17322

\title{
An example of degeneration on the noded Schottky space.
}

\author{
Rubén A. HIDALGO
}

\begin{abstract}
In these notes we construct explicit examples of degenerations on the noded Schottky space $\{7]$ of genus $g \geq 3$. The particularity of these degenerations is the invariance under the action of a dihedral group of order $2 g$. More precisely, we find a two-dimensional complex manifold in the Schottky space such that all groups (including the limit ones in the noded Schottky space) admit a fixed topological action of a dihedral group of order $2 g$ as conformal automorphisms.
\end{abstract}

\section{Preliminaries}

The basic literature for this section is [11]. A conformal automorphism of the Riemann sphere $\widehat{\mathbb{C}}$ is called a Möbius transformation and has the following form

$$
T(z)=\frac{a z+b}{c z+d}
$$

where $a, b, c, d \in \mathbb{C}$ and $a d-b c \neq 0$.

The set $\boldsymbol{M}$ consisting of all Möbius transformations is a topological group isomorphic to $\operatorname{PSL}(2, \mathbb{Q})$, in particular, we can talk about discrete groups of Möbius transformations.

A Kleinian group $G$ is a group of Möbius transformations for which there is a point $x \in \widehat{\mathbb{C}}$ satisfying the following:

1991 Mathematics Subject Classification: 30F10, 30F40.

This work was partially supported by projects Fondecyt 8970007 and UTFSM 971223.

Servicio Publicaciones Univ. Complutense. Madrid, 1998. 
(1) $G(x)=\{g \in G: g(x)=x\}$ is finite,

(2) there exists an open neighborhood $U$ of $x$ such that

(2.1) $g(U) \cap U=0$ for all $g \in G-G(x)$ and

(2.2) $g(U)=U$ for all $g \in G(x)$.

The set of points of the Riemann sphere for which the above properties hold is called the region of discontinuity of $G$ and denoted by $\Omega(G)$. Its complement $\Lambda(G)$ is called the limit set of $G$.

A Kleinian group is in particular discrete, but the converse is false. We are interested in a very particular type of Kleinian groups called noded Schottky groups, that is, geometrically finite (see the definition in 111) discrete groups of Möbius transformations isomorphic to a free group of finite rank (the rank is also called the genus of the group). In [7] we have observed that a noded Schottky group is in fact a Kleinian group.

Example of Noded Schottky groups are for instance:

(a) Schottky groups: Purely loxodromic Kleinian groups. These are the noded Schottky groups having no parabolic transformations (Möbius transformations having exactly one fixed point).

(b) Schottky-Type groups: These are Kleinian groups constructed from Klein-Maskit first combination theorem using cyclic groups of either loxodromic or parabolic type.

(c) Torsion-free finitely generated quasifuchsian groups with parabolics elements (either of second or first kind): These are quasifuchsian deformations of torsion-free finitely generated Fuchsian groups.

(d) Groups obtained combining the above ones using the Klein-Maskit Combination theorems.

In the above, we that the groups in category (a) belong to category (b), those of category (b) belong to category (c), and those in category (c) belong to category (d). It is interesting to note that there are more complicated noded Schottky groups than the above examples. 
A marked noded Schottky group of genus $g$ is by definition a $(g+1)$ tuple $\left(G, T_{1}, \ldots, T_{g}\right)$, where $G$ is a noded Schottky group of genus $g$, and $T_{1}, \ldots, T_{g}$ is a set of free generators of $G$.

It is not hard to prove that if we have a marked noded Schottky group of genus $g \geq 2$ as above, then we cannot have two different generators sharing a common fixed point (this will contradicts the discreteness property of Kleinian groups).

Given a noded Schottky group $G$ of genus $g$, we denote by $\Omega^{\text {cxt }}(G)$ its region of discontinuity, that is, $\Omega^{e x t}(G)=\Omega(G) \cup P(G)$, where $\Omega(G)$ is its region of discontinuity and $P(G)$ is the set of fixed points of the parabolic transformations of $G$. We consider on $\Omega^{\text {ext }}(G)$ the topology generated by the usual open sets of $\Omega(G)$ and the sets of the form $B_{1} \cup B_{2} \cup\{x\}$, where $x \in P(G)$ and $B_{1}$ and $B_{2}$ are disjoint open round discs inside $\Omega(G)$ both tangent at $x$. In this topology, the group $G$ acts as group of homeomorphisms, preserving each $\Omega(G)$ and $P(G)$, so that its restriction to $\Omega(G)$ is conformal. The quotient $S=\Omega^{e x t}(G) / G$ is a stable Riemann surface of genus $g$ (for $g=1$ we means at most one node). We say that the noded Schottky group $G$ uniformizes $S$ and that the natural map $Q: \Omega^{e x t}(G) \rightarrow S$ is the uniformizing map. Reciprocally, each stable Riemann surface of genus $g$ is uniformized by some noded Schottky group (see retrosection theorem with nodes in [7|).

In this work we are interested in finite normal extensions of noded Schottky groups, that is, stable Riemann surfaces with automorphisms that are reflected by noded Schottky groups. In the particular case of Schottky groups, finite normal extensions have been studied, for instance, in [8] and [10].

\section{A family of Möbius transformations and noded Schottky groups}

Let us consider for each integer $g \geq 3$ the Möbius transformations

$$
A_{g}(z)=e^{\frac{2 \pi i}{b}} z, \quad \text { and } \quad B(z)=\frac{1}{z} .
$$

The group $F_{g}$, generated by $A_{g}$ and $B$, is isomorphic to the dihedral group of order $2 g$, and

$$
A_{g}^{g}=B^{2}=\left(B A_{g}\right)^{2}=I .
$$


A fundamental domain for $F_{g}$ is given by the region $D_{g} \cup P_{g}$, where

$$
\begin{gathered}
D_{g}=\left\{z \in \mathbb{C}:|z|<1, \quad \frac{-\pi i}{g}<\arg (z) \leq \frac{\pi i}{g}\right\}, \text { and } \\
P_{g}=\left\{z \in \mathscr{C}:|z|=1, \quad 0 \leq \arg (z) \leq \frac{\pi i}{g}\right\} \cup\{0\} .
\end{gathered}
$$

Denote by $X$ the set of Möbius transformations $C \neq B$ such that $B C B=C^{-1}$ and $C(0) \neq 0$. Such a Möbius transformation $C \in X$ has the form

$$
C(z)=\frac{a z+1}{=z+b}, \quad a b+1 \neq 0 .
$$

If we consider the two-dimensional complex manifold

$$
W=\left\{(a, b) \in \mathbb{C}^{2}: a b+1 \neq 0\right\},
$$

then we have a bi-analytic map given by

$$
\phi: W \rightarrow X,(a, b) \rightarrow C(z)=\frac{a z+1}{-z+b} .
$$

For each $p=(a, b) \in W$, we consider the transformations

$$
D_{0}(p)=\phi(p), D_{i}(p)=A_{g}^{i} D_{0}(p) A_{g}^{-i}, \quad i \in\{1, \ldots, g-1\} .
$$

Denote by $G(p)$ the group generated by the above Möbius transformations. We also denote by $\mathcal{S}$ (respectively, $\mathcal{N S}$ ) the set of points $p \in W$ such that the group $G(p)$ is a Schottky [3] (respectively, noded Schottky [7]) group of genus $g$, free generated by the above transformations.

Since every Schottky group is in particular a noded Schottky [7], we have

$$
s \subset \mathcal{N S} \subset W .
$$

We have that $\mathcal{S}$ is an open and connected (consequence of quasiconformal deformation theory [1]) subset of $W$ and, in particular, a connected complex manifold of dimension two. Moreover, the closure of $\mathcal{S}$ in $W$ contains (strictly) $\mathcal{N} \mathcal{S}$.

Proposition 1. If $g$ is even, then $\mathcal{N S} \cap\{(a, b) \in W: a=b\}=\emptyset$.

Proof. f If we have $a=b$, then both $D_{0}(p)$ and $D_{\frac{g}{2}}(p)$ have fixed points $\pm i$. It follows that $G(p)$ cannot have these two transformations as free generators and to be discrete at the same time. 
If we denote by $\mathcal{S}_{g}$ (respectively, $\mathcal{N} \mathcal{S}_{g}$ ) the Schottky space (respectively, noded Schottky space [7]) of genus $g$, then we have a natural map

$$
\psi_{g}: \mathcal{N S} \rightarrow \mathcal{N} \mathcal{S}_{g}: p \rightarrow\left[\left(G(p), D_{0}(p), \ldots, D_{g-1}(p)\right)\right]
$$

Its restriction to the manifold $\mathcal{S}$ (into $\mathcal{S}_{g}$ ) is holomorphic.

Proposition 2. The map $\psi_{g}$ is two-to-one. Moreover, if $\psi_{g}(p)=\psi_{g}(q)$, then $q=-p$.

Proof. Let $p$ and $q$ two different points in $\mathcal{N S}$. The equality $\psi_{g}(p)=$ $\psi_{g}(q)$ asserts the existence of a Möbius transformation $H \neq I$ such that $B H^{-1} B H$ and $H^{-1} A_{g}^{-k} H A_{g}^{k}$ commute with $D_{0}(p)$, for all $k=1, \ldots, g-1$.

(1) Let us assume first that $H^{-1} A_{g}^{-k} H A_{g}^{k} \neq I$, for all $k=1, \ldots, g-1$. The fact that $D_{0}(p)$ is not elliptic of order two ensures that the commutativity conditions are equivalent to the equality of the fixed points of the transformations $D_{0}(p)$ and $H^{-1} A_{g}^{-k} H A_{g}^{k}$, for all $k=1, \ldots, g-1$.

The equation of fixed points of $D_{0}(p)$ is given by $t^{2}+(a-b) t+1=0$. It follows that the two fixed points of $D_{0}(p)$ are inverse of each other. In particular, if we write

$$
H(u)=\frac{x u+y}{z u+w} \text { and } \rho=e^{\frac{\pi i}{g}}
$$

then the above asserts the equality $w y\left(\rho^{-2 k}-1\right)=1$, for $k=1, \ldots, g-1$. Since $g \geq 3$, we have that this is impossible.

(2) If we have that $H^{-1} A^{-k} H A^{k}=I$ for some $k=1, \ldots, g-1$, then it follows that $H$ and $A$ commute. Since $A$ has no order two, it follows that $H$ fixes the points 0 and $\infty$, that is, $H(u)=\lambda u$. On the other hand, we now that $B H^{-1} B H$ must commute with $D_{0}(p)$. But $B H^{-1} B H(u)=$ $\lambda^{2} u$. If $\lambda^{2} \neq 1$, then the commutativity property and the fact that $D_{0}(p)$ is not elliptic of order two assert that the fixed points of $D_{0}(p)$ must be 0 and $\infty$, a contradiction. It follows then that $\lambda=\{ \pm 1\}$. Since we have assumed $H \neq I$, we have $H(u)=-u$ and, it follows that $q=-p$.

Let us consider the holomorphic involution $\eta: \widehat{\mathbb{C}}^{2} \rightarrow \widehat{\mathbb{C}}^{2}$ defined by $\eta(p)=-p$. Set $U$ the cyclic group generated by this involution. The set of fixed points of $\eta$ is just the singleton $\{(0,0)\}$. It follows that $W / U$ 
is a complex orbifold with only one singular point. If we consider the map $t: W \rightarrow \mathbb{C}^{2}$ defined by $t(z, w)=\left(z^{2}, z w\right)$, then $t(p)=t(q)$ if and only if $p= \pm q$. The image of $t$ is the the locus $\sigma^{2}-\{(t,-1): t \in \mathbb{G}\}$. In particular, $W / U$ is a complex manifold of dimension two.

The map $\psi_{g}$ then produces a one-to-one map from $\mathcal{N S} / U$ into $\mathcal{N} \mathcal{S}_{g}$, with holomorphic restriction to $S / U$.

Remark 1. For each $p \in W$, the group $K(p)$ generated by $G(p), A_{g}$ and $B$ is a finite normal extension of $G(p)$. In particular,

(1) $K(p)$ is Kleinian group if and only if $G(p)$ is Kleinian group.

(2) $K(p)$ is geometrically finite if and only if $G(p)$ is geometrically finite.

(3) The region of discontinuity of $G(p)$ and $K(p)$ are the same.

(4) $K(p) / G(p)$ is isomorphic to the dihedral group of order $2 g$.

\section{Hyperelliptic noded Schottky roups}

A (marked) noded Schottky group $\left(G, A_{1}, \ldots, A_{g}\right)$ of genus $g$ is called hyperelliptic if there is a Möbius transformation $H$ such that $H^{2}=I$ and $H A_{i} H=A_{i}^{-1}$, for all $i \in\{1, \ldots, g\}$.

We denote by $\mathcal{H N S} \mathcal{S}_{g}$ the locus in $\mathcal{N} \mathcal{S}_{g}$ consisting of hyperelliptic noded Schottky groups, and we set $\mathcal{H} \mathcal{S}_{g}=\mathcal{H N} \mathcal{S}_{g} \cap \mathcal{S}_{g}$ the set consisting of the hyperelliptic Schottky groups. It was shown in $[10]$ that $\mathcal{H} \mathcal{S}_{g}$ parameterizes all hyperelliptic Riemamn surfaces of genus $g$.

Set $\mathcal{H N S}=\psi_{g}^{-1}\left(\mathcal{H N S} \mathcal{S}_{g}\right)$ and $\mathcal{H S}=\psi_{g}^{-1}\left(\mathcal{H} \mathcal{S}_{g}\right)$.

We have a natural involution on $W$ defined by

$$
\tau: W \rightarrow W: \tau((a, b))=(b, a) .
$$

Proposition 3. If $g$ is even, then $\mathcal{H N S}=\emptyset$. If $g$ is odd, then $\mathcal{H N S}=$ $\{(a, b) \in \mathcal{N S}: a=b\}=\pi(F i x(\tau))$.

Proof. A point $p=(a, b) \in \mathcal{N} S$ belongs to $\mathcal{H N S}$ if and only if there is a Möbius transformation $H$ with $H^{2}=I$ and $H D_{i}(p) H=D_{i}(p)^{-1}$, for all $i$. 
The fixed points of $D_{i}(p)$ are $A_{g}^{i}(x)=\rho^{i} x$ and $A_{g}^{i}\left(\frac{1}{x}\right)=\frac{\rho^{i}}{x}$, where $\rho=e^{\frac{2 \pi i}{g}}$ and the fixed points of $D_{0}(p)$ are $x$ and $\frac{1}{x}$. It follows that

$$
H\left(\rho^{i} x\right)=\frac{\rho^{i}}{x}, \quad \text { for each } \quad i=0,1, \ldots, g-1 .
$$

Using the fact that $H(z)=\frac{A z+S}{T z-R}$, with $R^{2}+S T=-1$ and the above equation for $i=0,1,2$, we obtain (after some minor computations) that $H(z)=-z$. It follows that $x= \pm i$ and, in particular, $a=b$. As a consequence,

$$
\mathcal{H N S}=\{(a, b) \in \mathcal{N S}: a=b\}=\pi(\operatorname{Fix}(\tau)) .
$$

When $g$ is even we have, from proposition 1 , that $\mathcal{H N S}=\emptyset$.

The above says that, for $g$ odd, the hyperelliptic involution is represented in $W$ by the involution $\tau$.

Remark. It is known that there are hyperelliptic Riemann surfaces of genus $g$ having automorphism group isomorphic to a dihedral group of order $2 g$ for which the group generated by such a group together the hyperelliptic involution do not satisfy condition (A) of [8] as can be seen in [2] and [9], in particular, cannot be uniformized by Schottky groups.

\section{Boundary points of $\psi_{g}(\mathcal{S}) \subset \mathcal{N} \mathcal{S}_{g}$}

Now we proceed to look at the boundary points of the two-dimensional complex manifolds $\psi_{g}(\mathcal{S})$ in $\mathcal{N S}_{g}$. These points are exactly $\psi_{g}(\mathcal{N S})-$ $\psi_{g}(\mathcal{S})$.

Let us consider the following regions:

$$
\begin{aligned}
& \mathcal{R}_{1}=\left\{z \in \mathbb{C}: \arg (z) \in\left(\frac{-\pi}{g}, \frac{\pi}{g}\right), 0<|z|<1\right\} \\
& \mathcal{R}_{2}=\left\{z \in \mathbb{C}: \arg (z) \in\left(0, \frac{\pi}{g}\right)\right\} \\
& \mathcal{R}_{3}=\left\{z \in \mathbb{C}: \arg (z) \in\left(\frac{-\pi}{g}, \frac{\pi}{g}\right)\right\}
\end{aligned}
$$




$$
\mathcal{R}_{4}=\left\{z \in \mathbb{C}: \arg (z) \in\left(\frac{\pi}{2}-\frac{\pi}{g}, \frac{\pi}{2}+\frac{\pi}{g}\right)\right\}
$$

and the lines

$$
\begin{aligned}
& L_{1}=\left\{z \in \mathbb{C}: \arg (z)=\frac{\pi}{g}\right\} \\
& L_{2}=\left\{z \in \mathbb{C}: \arg (z)=\frac{-\pi}{g}\right\} \\
& L_{3}=\left\{z \in \mathbb{C}: \arg (z)=\frac{\pi}{2}+\frac{\pi}{g}\right\} \\
& L_{4}=\left\{z \in \mathbb{C}: \arg (z)=\frac{\pi}{2}-\frac{\pi}{g}\right\}
\end{aligned}
$$

Set $\theta_{*}(z)=\frac{1}{\bar{z}}$ the reflection on the unit circle.

If we consider a circle $C \subset \mathcal{R}_{3}$ such that $B(C) \cap C=\emptyset$ (or $C \subset \mathcal{R}_{4}$ for $g$ odd as we do in degeneration 4.6), and $\Theta$ denotes the reflection on $C$, then there exists a point $p \in \mathcal{S}$ such that $\theta_{*} \Theta=D_{0}(p)$. In fact, if $w$ is the center of such a circle and $r$ is radius, then $p=\left(\frac{-1}{w}, \frac{w^{2}-r^{2}}{w}\right)$.

In what follows, we proceed to approach the circle $C$ to a limit circle contained in the closure of the region $\mathcal{R}_{3}$ (or $\mathcal{R}_{4}$ for $g$ odd as done in degeneration 4.6). This procedure will produce points in $N S$ representing noded Riemann Schottky groups as limit of Schottky groups in $\mathcal{S}$.

\subsection{Degeneration of type 1}

Let us start with a circle $C \subset \mathcal{R}_{1}$ and, by moving both the center and the radius without getting out of the above region, we can obtain as limit a circle $C_{1}$ tangent to the unit circle at 1 and $C_{1}-\{1\} \subset \mathcal{R}_{1}$. Let $\Theta_{1}$ be the reflection of this limit circle.

In this situation, there is a point $p_{1} \in \mathcal{N S}-\mathcal{S}$ with $\Theta_{*} \Theta_{1}=D_{0}\left(p_{1}\right)$ a parabolic transformation with fixed point 1 . The group $G\left(p_{1}\right)$ is a noded Schottky group (in fact a Schottky-Type group) and it uniformizes (see [7]) a stable Riemann surface topologically equivalent to the one in figure 1. The locus in $\mathcal{N S}-\mathcal{S}$ obtained by this type of degeneration is given by

$$
\left\{(a, b) \in W: b=a+2, \quad-\left(1+\sin \left(\frac{\pi}{g}\right)\right)<a<-1\right\} .
$$




\subsection{Degeneration of type 2}

As is the above case, we can move the circle $C \subset \mathcal{R}_{1}$ to obtain a limit circle $C_{2}$ tangent to the lines $L_{1}$ and $L_{2}$, and contained in $\mathcal{R}_{1}$ (by exception of the tangencies). If $\Theta_{2}$ denotes the reflection on this circle, then there is a point $p_{2} \in \mathcal{N S}-\mathcal{S}$ with $\Theta_{*} \Theta_{2}=D_{0}\left(p_{2}\right)$ a loxodromic transformation such that $A_{g}^{-1} D_{0}\left(p_{2}\right)^{-1} A_{g} D_{0}\left(p_{2}\right)$ is parabolic with fixed point $C_{2} \cap L_{1}$. The group $G\left(P_{2}\right)$ is a noded Schottky group obtained from the free combination of two torsion-free finitely generated Fuchsian groups uniformizing spheres with punctures. This group uniformizes a stable Riemann surface topologically equivalent to the one of figure 2 . The locus in $\mathcal{N S}-\mathcal{S}$ obtained by this type of degeneration is given by

$$
\left\{(a, b) \in W: a b=-\cos ^{2}\left(\frac{\pi}{g}\right), \quad a<-\left(1+\sin \left(\frac{\pi}{g}\right)\right)\right\} .
$$

\subsection{Degeneration of type 3}

Combining degenerations 1 and 2, we can obtain a limit circle $C_{3}$ tangent to the lines $L_{1}, L_{2}$ and the unit circle. If $\theta_{3}$ denotes the reflection on such a circle, then there is a point $p_{3} \in \mathcal{N S}-\mathcal{S}$ with $\Theta_{*} \Theta_{3}=D_{0}\left(p_{3}\right)$ a parabolic transformation with fxed point 1 such that $A_{g}^{-1} D_{0}\left(p_{3}\right)^{-1} A_{g} D_{0}\left(p_{3}\right)$ is parabolic with fixed point $C_{3} \cap L_{1}=e^{\frac{\pi i}{g} \frac{\cos \frac{\pi}{g}}{1+\sin \frac{\pi}{g}}}$. In this case, the center of $C_{3}$ is $\frac{1}{1+\sin \left(\frac{\pi}{g}\right)}$ and its radius is $\frac{\sin \left(\frac{\pi}{g}\right)}{1+\sin \left(\frac{\pi}{g}\right)}$. It follows that

$$
p_{3}=\left(-1-\sin \left(\frac{\pi}{g}\right), \frac{\cos ^{2}\left(\frac{\pi}{g}\right)}{1+\sin \left(\frac{\pi}{g}\right)}\right) .
$$

The group $G\left(p_{3}\right)$ uniformizes a stable Riemann surface topologically equivalent to the one described in figure 3.

\subsection{Degeneration of type 4}

We can start with a circle $C \subset \mathcal{R}_{2}$ and move it in this region to obtain a limit circle $C_{4}$ tangent to $L_{1}$ at $e^{\frac{n i}{g}}$, and contained in the above region. If $\Theta_{4}$ denotes the reflection on $C_{4}$, then there is a point $p_{4} \in \mathcal{N}-\mathcal{S}$ with $B \Theta_{*} \Theta_{4}=D_{0}\left(p_{4}\right)$ a loxodromic transformation such that $A_{g} D_{0}\left(p_{4}\right)$ 
is parabolic with fixed point $e^{\frac{\pi i}{g}}$. This group $G\left(p_{4}\right)$ is a torsion-free Fuchsian group of the second kind acting on the unit disc $\boldsymbol{H}$, freely generated by $g$ hyperbolic transformations with the product of them a parabolic, with $H / G\left(p_{4}\right)$ a sphere with $g$ holes and one puncture. In particular, it is a noded Schottky group uniformizing a stable Riemann surface topologically equivalent to the one of the figure 4 . In this case, the locus in $\mathcal{N S}-\mathcal{S}$ obtained by this type of degeneration is given by

$$
\begin{gathered}
\left\{(a, b) \in W: a=-\frac{\cos \left(\frac{\pi}{g}-\theta\right)}{e^{i \theta}}, \quad b=\frac{e^{i \theta} \tan ^{2}\left(\frac{\pi}{g}-\theta\right)+\cos \left(\frac{\pi}{g}-\theta\right)}{\cos \left(\frac{\pi}{g}-\theta\right)},\right. \\
\left.\frac{\pi}{2 g}<\theta<\frac{\pi}{g}\right\} .
\end{gathered}
$$

\subsection{Degeneration of type 5}

As before, we can obtain a limit circle $C_{5}$, tangent to $L_{1}$ at $e^{\frac{\pi i}{g}}$, and tangent to the real axis at 1 . If $\Theta_{5}$ denotes the reflection on $C_{5}$, then there is a point $p_{5} \in \mathcal{N S}-\mathcal{S}$ with $B \theta_{4} \Theta_{5}=D_{0}\left(p_{5}\right)$ a parabolic transformation fixing 1 such that $A_{g} D_{0}\left(p_{5}\right)$ is parabolic with fixed point $e^{\frac{\pi i}{g}}$. The group $G\left(p_{5}\right)$ is a torsion-free Fuchsian group freely generated by $g$ parabolic transformations with the product of them also parabolic, acting on the unit disc $\boldsymbol{H}$ so that $\boldsymbol{H} / G\left(p_{5}\right)$ is an sphere with $(g+1)$ punctures. In particular, it is a noded Schottky group that uniformizes a stable Riemann surface as described in figure 5 . We also have

$$
p_{5}=\left(-\frac{\cos \left(\frac{\pi}{2 g}\right)}{e^{\frac{\pi i}{2 g}}},-\frac{e^{\frac{\pi i}{2 g}} \tan ^{2}\left(\frac{\pi}{2 g}\right)+\cos \left(\frac{\pi}{2 g}\right)}{\cos \left(\frac{\pi}{2 g}\right)}\right)
$$

\subsection{Degeneration of type 6}

The next type of degenerations only work for $g$ odd. We assume then $g \geq 3$ and odd. Let $r>0$ and $w>0$ be such that the circle $C$ centered at $i w$ and radius $r$ is contained in the region $\mathcal{R}_{1}$. Let $\theta$ be the reflection on $C, \hat{J}(z)=-z$ and $J(z)=-z+2 i w$.

We consider the transformation $\widehat{J} \theta_{*}, \exists=D_{0}(p)$, where

$$
p=\left(\frac{-1}{i w}, \frac{-\left(w^{2}+r^{2}\right)}{i w}\right) \in \mathcal{W} .
$$


The family of circles obtained by rotation of $C$ by powers of $A_{g}$ are disjoint if and only if $r<w \sin \left(\frac{\pi}{g}\right)$. If $r=w \sin \left(\frac{\pi}{g}\right)$, then they are tangent.

The circle $C$ is disjoint from the family of circles obtained by rotations of $B(C)=D_{0}(p)(C)$ if and only if $w^{4}-2\left(r^{2}+\cos \left(\frac{\pi}{g}\right)\right) w^{2}+\left(r^{2}-1\right)^{2}>$ 0 . Equality to zero is equivalent to tangency.

It follows that $p \in \mathcal{S}$ if both inequalities above are satisfied at the same time. We may try to approach to some points in $\mathcal{N S}-\mathcal{S}$ by making some of the above inequalities an equality. In fact, taking $r=w \sin \left(\frac{\pi}{g}\right)$ and $w^{4}-2\left(r^{2}+\cos \left(\frac{\pi}{g}\right)\right) w^{2}+\left(r^{2}-1\right)^{2}>0$, we obtain the same type of noded Schottky groups obtained in degeneration 2. In this case, $p=\left(\frac{-1}{i w}, i w\left(1+\sin ^{2}\left(\frac{\pi}{g}\right)\right)\right)$.

The another possibility is $r<w \sin \left(\frac{\pi}{g}\right)$ and $w^{4}-2\left(r^{2}+\cos \left(\frac{\pi}{g}\right)\right) w^{2}+$ $\left(r^{2}-1\right)^{2}=0$. In this case, we obtain a point $p_{6} \in \mathcal{W}$ so that the group $G\left(p_{6}\right)$ is a quasifuchsian group of the first kind uniformizing a surface of signature $\left(\frac{g-1}{2}, 2 ; \infty, \infty\right)$ and, in particular, a noded Schottky group with $p_{6} \in \mathcal{N S}-\mathcal{S}$. Figure 6 shows the topological type of the stable Riemann surface $\Omega^{\operatorname{ext}}\left(G\left(p_{6}\right)\right) / G\left(p_{6}\right)$. the locus in $\mathcal{N S}-\mathcal{S}$ obtained by this degeneration is given by

$$
\begin{gathered}
\left\{\left(\frac{-1}{i w}, \frac{-\left(r^{2}+w^{2}\right)}{i w}\right) \in \mathcal{W}: w^{4}-2\left(r^{2}+\cos \left(\frac{\pi}{g}\right)\right) w^{2}+\left(r^{2}-1\right)^{2}=0,\right. \\
\left.r<w \sin \left(\frac{\pi}{g}\right)\right\} .
\end{gathered}
$$

For instance, if $w=1$, then $r$ is the positive root of $r^{4}-4 r^{2}+2(1-$ $\left.\cos \left(\frac{\pi}{g}\right)\right)=0$ with $r<1$, that is, $p_{6}=\left(i, i\left(3-\sqrt{2+2 \cos \left(\frac{\pi}{g}\right)}\right)\right)$. Another particular case in this degeneration is to assume $w^{2}+r^{2}=1$. This gives us a point $p h_{6} \in \mathcal{H N S}-\mathcal{H S}$ (the hyperelliptic involution is represented by $H(z)=-z)$. In this case, $w=\sqrt{\frac{1+\cos \left(\frac{\pi}{Q}\right)}{2}}$.

The group generated by $G\left(p h_{6}\right)$ and $H$ uniformizes an stable Riemann surface as described in figure 7 .

Question: Are all points in $\psi_{g}(\mathcal{N S})-\psi_{g}(\mathcal{S})$ described by the above degenerations? 
From the viewpoint of algebraic curves and principally polarized abelian varieties, some explicit degenerations have been constructed in $[4],[5]$ and $[6]$.

Remark 2. The sublocus $\mathcal{N} R=\{(a, b) \in \mathcal{N} \mathcal{S}: a, b \in \mathbb{R}\}$ represents noded Schottky groups admitting the symmetry $\theta_{*}$. We also have that $\mathcal{N R} \cap \mathcal{H N S}=\{(a, b) \in \mathcal{N} \mathcal{S}: a=b \in \mathbb{R}\}$.

Remark 3. The ideas carried out for the group $F_{g}$ can be used for any finite Kleinian group to construct degenerations on the noded Schottky space.

Acknowledge. The author would like to thank the referee for the comments and specially for pointing out a mistake in the original statement of Proposition 2. 


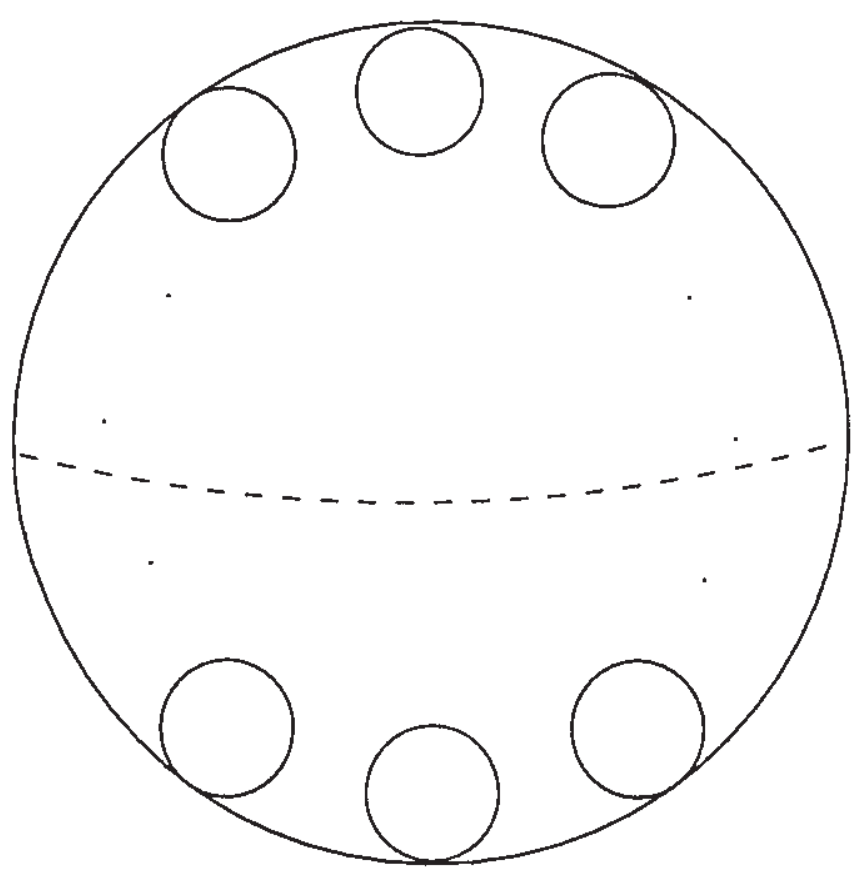

A stable Riemann surface of genus $g$ with exactly one component and $g$ nodes.

Figure 1. 


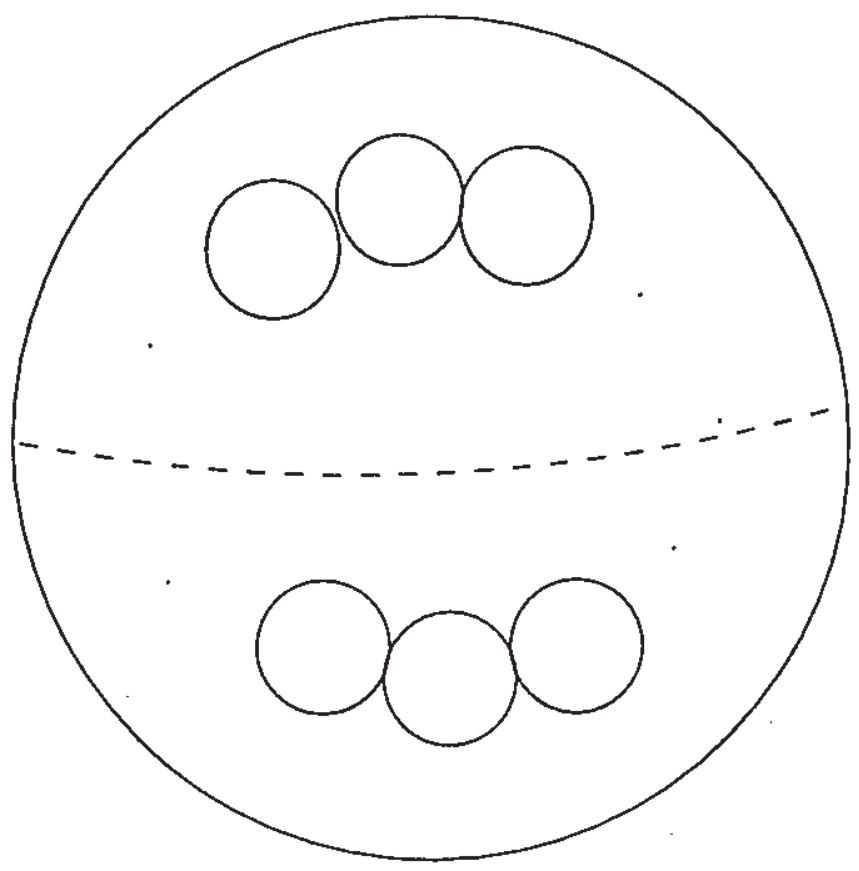

A stable surface of genus $g$ with exactly two components and $g$ nodes. One component is a $g$-pointed sphere and the other is a $g$-pointed torus.

Figure 2. 


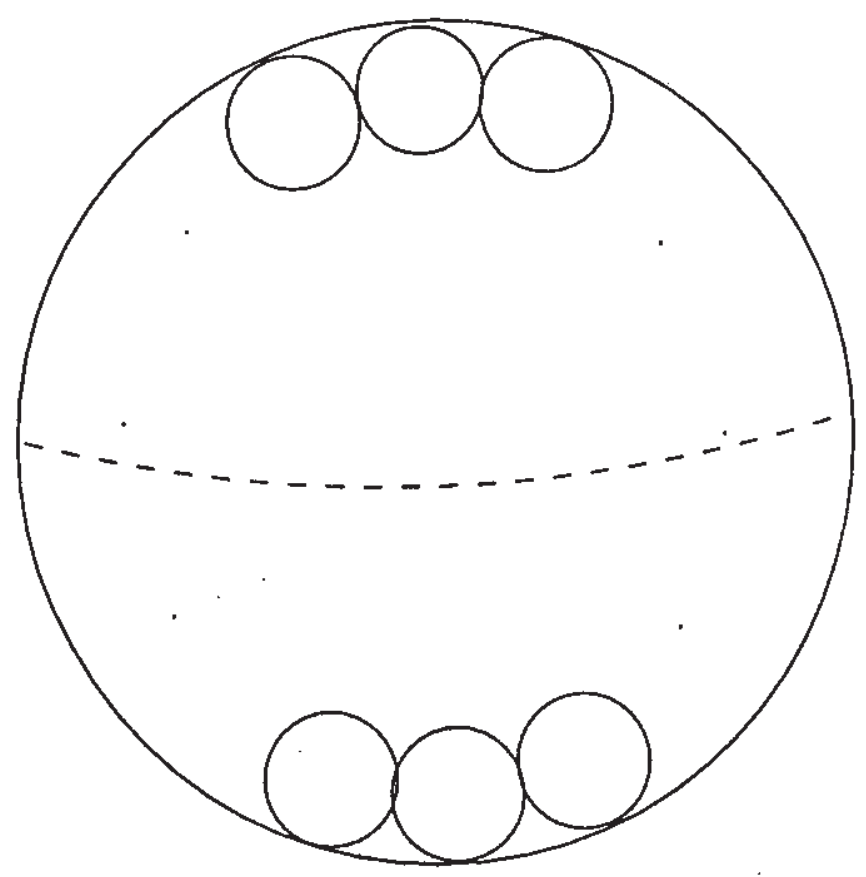

An stable Riemann surface of genus $g$ with exactly $(1+g)$ components. One of the components is a $g$-pointed sphere and the other are threepointed spheres.

Figure 3. 


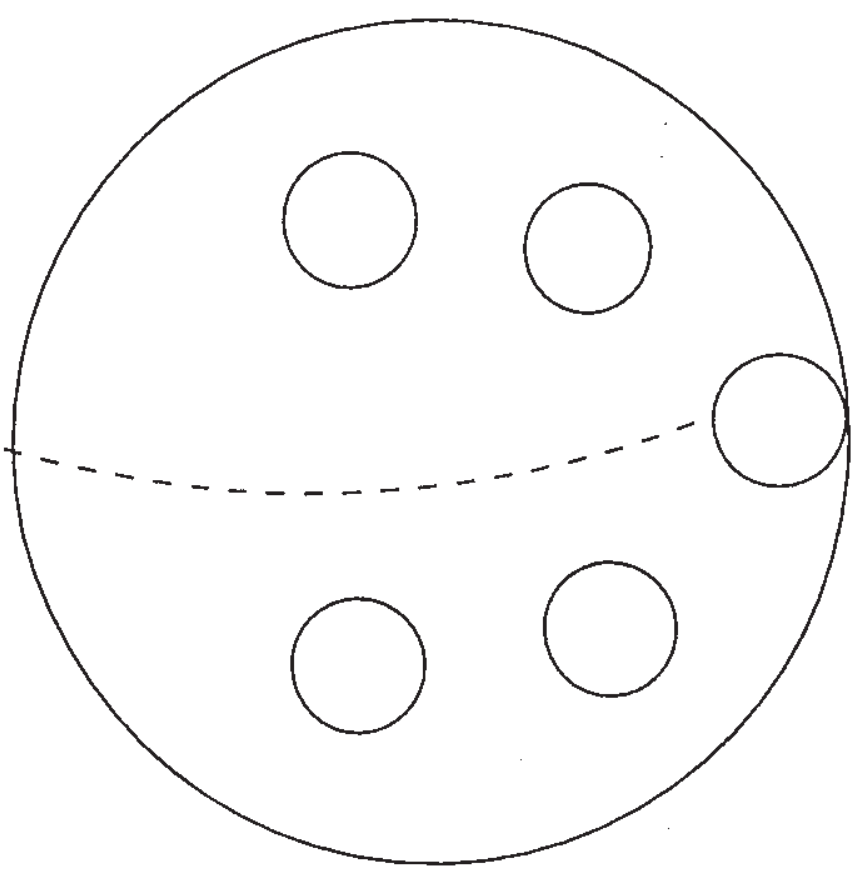

A stable Riemann surface of genus $g$ with exactly one components and one node. The component is a two-pointed Riemann surface of genus $(g-1)$.

Figure 4. 




A stable Riemann surface of genus $g$ with exactly two components and $(g+1)$ nodes. Each component is a $(g+1)$-pointed sphere.

Figure 5. 


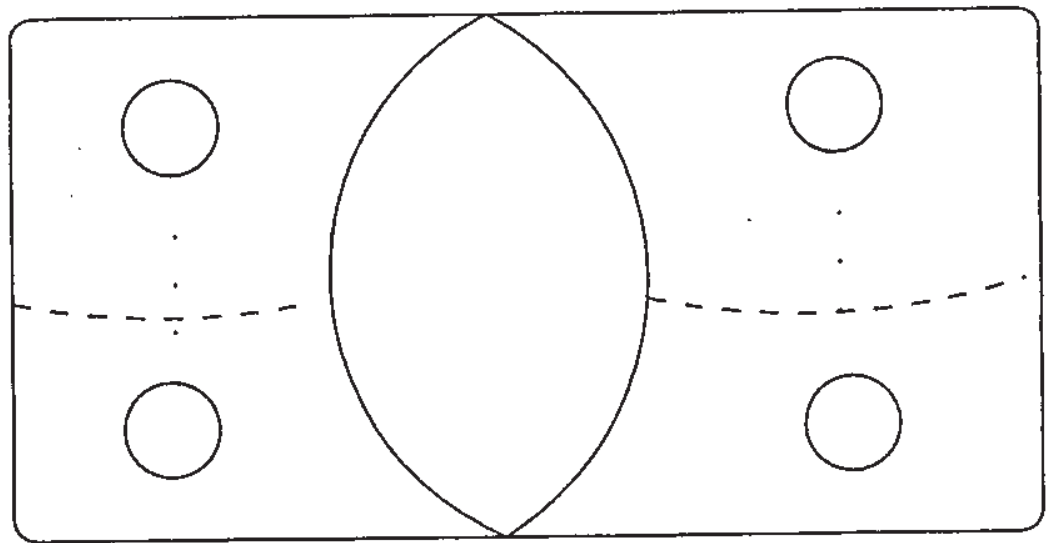

A stable Riemann surface of genus $g$ with two components and two nodes. Each component is a two-pointed Riemann surface of genus ( $g-$ 1) $/ 2$.

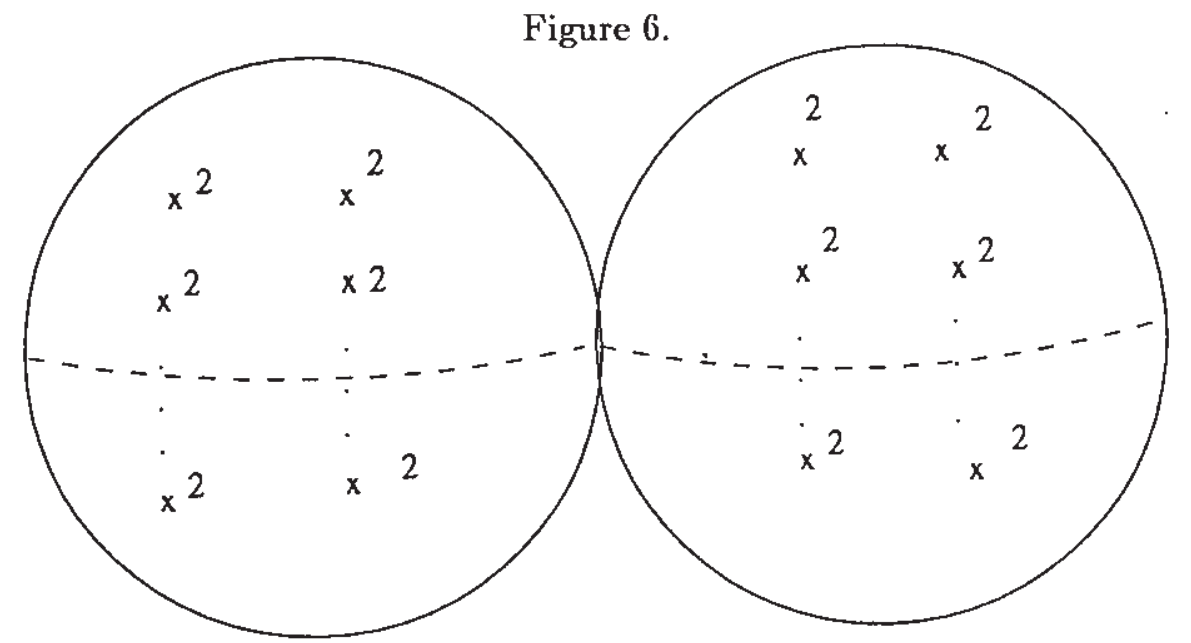

'Two Riemann spheres touching at one node. Each component is a Riemaniu sphere with $(g+1)$ points of order two.

Figure 7. 


\section{References}

[1] L. Bers. Uniformization by Beltrami equations, Comm. Pure Appl. Math. 14 (1961), 215-228.

[2] R. Brandt and H. Stichtenoth. Die Automorphismengruppen Hyperelliptischer Kurven, Manuscripta Math. 55 (1986), 83-92.

[3] V. Chuckrow. On Schottky groups with applications to Kleinian groups, Ann. of math. 88 (1968), 47-61.

[4] V. González and R. Rodríguez. A pencil in $\widetilde{\mathcal{M}}_{6}$ with three points at the boundary, Geometria Dedicata 42 (1992), 255-265.

[5] Fermat's quartic curve, Klein's curve and the tetrahedron, Contemporary Mathematics, 201, (1996), 43-62.

[6] ___ On principally polarized Abelian varieties associated to Prisms and Pyramids, Seminario Geometria Compleja 3, Universidad Técnica Federico Santa María.

[7] R.A. Hidalgo. The noded Schottky space, Proc. of the London Math. Soc. 73 (1996), 385-403.

[8] _ـ On Schottky groups with automorphisms, Ann. Acad. Scie. Fenn. Ser. AI Mathematica 19 (1994), 259-289.

[9] ___ Closed Riemann surfaces with dihedral groups of automorphisms, Revista Proyecciones, Universidad Católica del Norte, 15 (1996), 47-90.

[10] L. Keen. On hyperelliptic Schottky groups, Ann. Acad. Sci. Fenn. Ser. AI Math. 5 (1980), 165-174.

[11] B. Maskit. Kleinian Groups, Grundlehren Math. Wiss. 287, Springer-Verlag, 1988.

Rubén A. Hidalgo

Departamento de Matemáticas Universidad Técnica Federico Santa María

Casilla 110-V, Valparaiso, Chile Recibido: 27 de Mayo de 1997

e-mail: rhidalgoomat.utfsm.c1

Revisado: 10 de Julio de 1997 